九州大学学術情報リポジトリ

Kyushu University Institutional Repository

\title{
MULTI-VALUED OPTIMAL STOPPING PROBLEM WITH MONOTONICITY
}

Ohtsubo, Yoshio

Department of Mathematics, Faculty of Science, Kochi University

https://doi.org/10.5109/13486

出版情報: Bulletin of informatics and cybernetics. 31 (2), pp.137-146, 1999-12. Research Association of Statistical Sciences

バージョン :

権利関係 : 


\title{
MULTI-VALUED OPTIMAL STOPPING PROBLEM WITH MONOTONICITY*
}

\author{
By
}

\author{
Yoshio OHTsubo ${ }^{\dagger}$
}

\begin{abstract}
We consider a multi-valued optimal stopping problem for indexed random sequences. We perform a scalarization of the random sequences and give characterization of optimal value process for scalarized problem. We also find optinal stopping time in the sense of Pareto optimality for original prablem. We apply its result to monotone cases; in which it is given an explicit optimal stopping time. It is given sufficient conditions for our problem to be a monotone case. One of conditions is a kind of concavity with regard to time parameter.
\end{abstract}

\section{Introduction}

We often happen to meet optimization problem with a lot of objectives. In this paper we formulate such a problem as optimal stopping problem for indexed random sequences and give a solution to the problem.

Let $(\Omega, \mathcal{F}, P)$ be a probability space and $\left(\mathcal{F}_{n}\right)_{n \in N}$ a filtration of $\mathcal{F}$; where $N=$ $\{0,1,2, \cdots\}$ is a discrete time space. Let $T$ be an index set of objects in our problem. For each $t$ in the index set $T$, let $\left(X_{n}^{t}\right)_{n \in N}$ be a random sequence defined on $(\Omega, \mathcal{F}, P)$ and adapted to $\left(\mathcal{F}_{n}\right)$ such that random variables $\sup _{n \in N} \sup _{t \in T}\left(X_{n}^{t}\right)^{+}$and $\sup _{t \in T}\left(X_{n}^{t}\right)^{-}$ are integrable with regard to $P$, where $x^{+}=\max (0, x)$ and $x^{-}=(-x)^{+}$. We assume that the set $\cup_{t \in T_{1}} B_{t}$ is $\mathcal{F}_{n}$ - measurable for any subset $T_{1}$ of $T$ and for any family $\left\{B_{t}: t \in T_{1}\right\}$ of $\mathcal{F}_{n}$-measurable sets. This assumption is clearly satisfied if $T$ is a countable set. For each $n \in N$, we also denote by $\Lambda_{n}$ the class of $\left(\mathcal{F}_{n}\right)$-stopping times $\tau$ such that $n \leq \tau<\infty$ almost surely.

Our aim is to maximize the expectation $E\left[X_{\tau}^{t}\right]$ with respect to $\tau$ in $\Lambda_{0}$ for every $t \in T$. Especially, when the index set $T$ consists of an element it is the classical optimal stopping problem (cf. Chow et al.(1971)), and when $T=\{1,2, \ldots, p\}$ it is a multiobjective stopping problem (cf. Gugerli(1987), Ohtsubo(1997)).

To accomplish our aim, we shall generalize the concept of Pareto optimality (cf. Aubin(1979, p.295), Yu(1985, p.22)). A Pareto optimal solution is also called an efficient

* This work was partly supported by Grant-in-Aid for Scientific Research C-2-10640124 from Ministry of Education, Science. Sports and Culture

$\ddagger$ Department of Mathematics, Faculty of Science, Kochi University: Kochi 780-8520, Japan

e-mail: ohtsubo@math.kochi-u.ac.jp 
or noninferior solution in the literature (see, for example. Yu(1985)). For the sake of general discussion we define a conditional expectation of $X_{\tau}^{t}$ at $n \in X$ by

$$
G_{n}(\tau ; t)=E\left[X_{\tau}^{t} \mid \mathcal{F}_{n}\right]
$$

for each $t \in T$ and $\tau \in \Lambda_{n}$. For $n \in N, \varepsilon \geq 0$ and probability measure $\mu$ on $\left(T, 2^{T}\right)$, we say that a stopping time $\tau_{\varepsilon}$ in $\lambda_{n}$ is $\left(\varepsilon_{,}, \mu\right)$-Pareto optimal at $n$, if there exists no stopping time $T$ in $A_{n}$ such that almost surely

$$
G_{n}(\tau ; t) \geq G_{n}\left(\tau_{s} ; t\right)+\varepsilon
$$

for every $t \in T_{0}$ and

$$
G_{n}(\tau ; t)>G_{n}\left(\tau_{\varepsilon} ; t\right)+\varepsilon
$$

for every $t \in T_{1}$, where $T_{0}$ and $T_{1}$ are some subsets of $T$ satisfying $\mu\left(T_{0}\right)=1$ and $\mu\left(T_{1}\right)>0$.

When $T$ is a finite set $\left\{t_{1}, t_{2}, \ldots, t_{N}\right\}$; and ne define $\mu$ to be probability measure such that $\mu\left(t_{k}\right)>0$ for every $k,(0, \mu)$-Pareto optimal stopping time $\tau_{\varepsilon}$ at $n$ satisfies the following condition: there exists no stopping time $\tau$ in $\Lambda_{n}$ such that almost surely

$$
G_{n}(\tau ; t) \geq G_{n}\left(\tau_{s} ; t\right)
$$

for every $t \in T$ and

$$
G_{n}(\tau ; t)>G_{n}\left(\tau_{\bar{t}} ; t\right)
$$

for some $t \in T$. This means well-known Pareto optimal solution in mathematical programming (cf. Aubin(1979), Yu(1985)).

Similarly we shall define weak Pareto optimal stopping time as follows : For $n \in N$, $\varepsilon \geq 0$ and probability measure $\mu$ on $\left(T, 2^{T}\right)$, it is said for a stopping time $\tau_{s}$ in $\Lambda_{n}$ to be $(\varepsilon, \mu)$-weak Pareto optimal at $n$, if there exists no stopping time $\tau$ in $\Lambda_{n}$ such that almost surely

$$
G_{n}(\tau ; t)>G_{n}\left(\tau_{s} ; t\right)+\varepsilon
$$

for every $t \in T_{0}$, where $T_{0}$ is some subset of $T$ satisfying $\mu\left(T_{0}\right)=1$.

We easily see that if $\tau_{\varepsilon}$ is $(\varepsilon, \mu)$-Pareto optimal at $n$, it is $(\varepsilon, \mu)$-weak Pareto optimal at $n$. In this paper we shall investigate only $(\varepsilon, \mu)$-Pareto optimal stopping time.

For the sake of simplicity, without further comments we assume that all inequalities and equalities between random variables hold in the sense of "almost surely".

In the second section, we consider a scalarization of our problem, give fundamental properties of optimal value process for the scalarized problem and find an optimal stopping time on the scalarized problem. By the results of the scalarization we find an $(\varepsilon, \mu)$-Pareto optimal stopping time for the original problem. In the third section, we consider the classical monotone case on the scalarized problem as a special model and we prove that a one-step-look-ahead (OLA) stopping time is $(0, \mu)$-Pareto optimal. It is given sufficient conditions for the problem to be a monotone case. The first condition is a kind of concavity with regard to time parameter on the original process and the second is a generalization of classical stopping problem. Finally in Section 4 we give 
examples of monotone case, including economic model which is a withdrawal problem from a market.

Such a problem is a generalization of multi-objective stopping problem, which has been investigated in Hisano(1980). Gugerli(1987) and Ohtsubo(1997). Hisano has introduce a partial order defined by a convex cone and give an existence theorem of optimal stopping time with regard to the order. Gugerli has considered such a problem for the class of all randomized stopping times on discrete time Markov chain and has characterized the set of all Pareto optimal stopping times. Ohtsubo(1997) has given a sufficient condition for multi-objective stopping problem to be a monotone case, and find 0-Pareto optimal stopping time which is OLA rule. This paper is not only a generalization of Ohtsubo(1997) but also contains many useful examples. In Stadje(1980), Preater(1993) and Gnedin(1994), multi-criteria best-choice problems have been investigated in different forms.

\section{Scalarization and Pareto Optimality}

In this section, we consider a scalarization of our problem with regard to probability measure on the set $T$, give fundamental properties of optimal value process for the scalarized problem and find an Pareto optimal stopping times on the original problem.

Let $\mathcal{M}$ denote the set of probability measures $\mu$ on the measurable space $\left(T, 2^{T}\right)$. This $\mu$ is called weighting function in mathematical programming. For $\mu$ in $\mathcal{M}$, we define a scalarized reward process $\left(X_{n}(\mu)\right)$ by

$$
X_{n}(\mu)=\int_{T} X_{n}^{t} d \mu(t),
$$

where $X_{n}^{t}$ is integrable from the conditions given in Section 1, and define the conditional expectation by

$$
G_{n}(\tau ; \mu)=\int_{T} G_{n}(\tau ; t) d \mu(t) \equiv E\left[X_{\tau}(\mu) \mid \mathcal{F}_{n}\right] .
$$

The last equality is obtained from the boundedness (from above) of $X_{\tau}^{t}$ on $\Omega \times T$ and from Fubini's theorem for conditional expectation. The optimal value process $\left(V_{n}(\mu)\right)$ for the scalarized problem is defined by

$$
V_{n}(\mu)=\underset{\tau \in \Lambda_{n}}{\operatorname{ess} \sup _{n}} G_{n}(\tau ; \mu), \quad n \in N^{+}
$$

for a given $\mu \in \mathcal{M}$.

For each $n \in N, \varepsilon \geq 0$ and $\mu \in \mathcal{M}$, we define a stopping time $\tau_{n}^{\varepsilon}(\mu)$ by

$$
\tau_{n}^{\varepsilon}(\mu)=\inf \left\{k \geq n \mid V_{k}(\mu) \leq X_{k}(\mu)+\varepsilon\right\},
$$

where inf $\phi=+\infty$.

The scalarized problem is one of classical stopping problems and hence it follows the theorem below, the proof of which refers to Theorems $4.1,4.2$ and 4.3 in Chow et al.(1971) or Proposition VI-1-2 and VI-1-3 in Neveu(1975). 
Proposition 2.1. Let $\mu$ in $\mathcal{M}$ be arbitraty.

(i) The optimal value process $T^{-}(\mu)=\left(I_{n}(\mu)\right)$ satisfies the following recursive relation:

$$
V_{n}(\mu)=\max \left(X_{n}(\mu), E\left[V_{n+1}(\mu) \mid \mathcal{F}_{n}\right]\right), \quad n \in N .
$$

(ii) $V(\mu)$ is the smallest supermartingale dominating the process $X(\mu)=\left(X_{n}(\mu)\right)$.

(iii) $\lim \sup _{n} V_{n}(\mu)=\lim \sup _{n} X_{n}(\mu)$.

(iv) For every $n \in N$ any $\varepsilon>0$, the stopping time $\tau_{n}^{*}(\mu)$ is $(\varepsilon, V(\mu))$-optimal at $n$ in the sense that $V_{n}(\mu) \leq G_{n}\left(\tau_{n}^{*}(\mu) ; \mu\right)+\varepsilon$. If the stopping time $\tau_{n}^{0}(\mu)$ is a.s. finite, $\tau_{n}^{0}(\mu)$ is $(0, V(\mu))$-optimal at $n$.

The general lemma below is an important result and an optimal stopping version of a well-known result in multi-objective problem on mathematical programming (cf. Proposition 3 in Aubin(1979, p.297)).

LEMMA 2.2. Let $n \in N, \varepsilon \geq 0$ and $\mu \in \mathcal{M}$ be arbitrary. If a stopping time $\tau_{\varepsilon}$ in $\Lambda_{n}$ satisfies inequality $V_{n}(\mu) \leq G_{n}\left(\tau_{s} ; \mu\right)+\varepsilon$, then $\tau_{\xi}$ is $\left(\varepsilon_{;}, \mu\right)$-Pareto optimal at $n$.

Proof. We suppose that the stopping time $\tau_{\xi}$ is not $\left(\varepsilon_{,} \mu\right)$-Pareto optimal at $n$. Then there exists a stopping time $\tau$ in $A_{n}$ such that $G_{n}(\tau ; t) \geq G_{n}\left(\tau_{z}: t\right)+\varepsilon$ for every $t \in T_{0}$ and $G_{n}(\tau ; t)>G_{n}\left(\tau_{\varepsilon} ; t\right)+\varepsilon$ for every $t \in T_{1}$ where $T_{0}$ and $T_{1}$ satisfy $\mu\left(T_{0}\right)=1$ and $\mu\left(T_{1}\right)>0$. Thus since $\left(T-T_{1}\right) \cap T_{0} \subset T_{0}, \mu\left(T-\left(T_{0} \cup T_{1}\right)\right)=0$ and $\mu\left(T_{0} \cup T_{1}\right)=1$, we have

$$
\begin{aligned}
G_{n}(\tau ; \mu) & =\int_{T} G_{n}(\tau ; t) d \mu(t) \\
& =\int_{T_{1}} G_{n}(\tau ; t) d \mu(t)+\int_{\left(T-T_{1}\right) \cap T_{0}} G_{n}(\tau ; t) d \mu(t)+\int_{T-\left(T_{1} \cup T_{L}\right)} G_{n}(\tau ; t) d \mu(t) \\
& >\int_{T} G_{n}\left(\tau_{\varepsilon} ; t\right) d \mu(t)+\varepsilon \mu\left(T_{0} \cup T_{1}\right) \\
& =G_{n}\left(\tau_{\varepsilon} ; \mu\right)+\varepsilon
\end{aligned}
$$

and hence $V_{n}(\mu)>G_{n}\left(\tau_{\varepsilon} ; \mu\right)+\varepsilon$, which is a contradiction. Hence the stopping time $\tau_{\varepsilon}$ is $(\varepsilon, \mu)$-Pareto optimal at $n$.

Proposition 2.1 and Lemma 2.2 immediately imply the following theorem.

Theorem 2.3. Let $n \in N^{*}$ and $\mu \in \mathcal{M}$ be arbitrary.

(i) For each $\varepsilon>0$, the stopping time $\tau_{n}^{\varepsilon}(\mu)$ is $\left(\varepsilon_{-} \mu\right)$-Pareto optimal at $n$.

(ii) If the stopping time $\tau_{n}^{0}(\mu)$ is a.s. finite, $\tau_{n}^{0}(\mu)$ is $(0, \mu)$-Pareto optimal at $n$. 


\section{Monotone Case}

For the scalarized reward process $\left(X_{n}(\mu)\right)$ defined in Section 2 where $\mu \in \mathcal{M}$, we define subsets $A_{n}(\mu)$ of $\Omega$ by

$$
A_{n}(\mu)=\left\{X_{n}(\mu) \geq E\left[X_{n+1}(\mu) \mid \mathcal{F}_{n}\right]\right\}: \quad n \in N,
$$

and define a stopping time $\sigma_{n}(\mu)$ by

$$
\sigma_{n}(\mu)=\inf \left\{k \geq n \mid X_{k}(\mu) \geq E\left[X_{k+1}(\mu) \mid \mathcal{F}_{k}\right]\right\}, \quad n \in N,
$$

that is,

$$
\sigma_{n}(\mu)(\omega)=\inf \left\{k \geq n \mid \omega \in A_{k}(\mu)\right\}, \quad \omega \in \Omega, n \in N,
$$

where inf $\phi=+\infty . \sigma_{n}(\mu)$ is called one-step-look-ahead (OLA) rule or myopic rule.

For each $\mu$ in $\mathcal{U}$, we introduce the following condition :

Condition $C(\mu)$. For every $n \in N, A_{n}(\mu) \subset A_{n+1}(\mu)$ and $\lim _{n \rightarrow \infty} P\left(A_{n}(\mu)\right)=1$.

When the condition $C(\mu)$ is satisfied for a given $\mu \in \mathcal{M}$, the scalarized stopping problem corresponding to $\mu$ is in a well-known monotone case (cf. Chow et al.(1971, p.54)).

Theorem 3.1. Suppose that Condition $C(\mu)$ is satisfied for a given $\mu$ in $\mathcal{M}$. Then for each $n \in N \sigma_{n}(\mu)$ is a.s. equal to $\tau_{n}^{0}(\mu)$ and is a.s. finite, and hence $\sigma_{n}(\mu)$ is $(0, \mu)-$ Pareto optimal at $n$.

PRoof. The first part : $\sigma_{n}(\mu)=\tau_{n}^{0}(\mu)<\infty$ a.s. is proved similarly to Theorem 3.3 in Chow et al.(1971). Hence Theorem 2.3 implies that $\sigma_{n}(\mu)$ is $(0, \mu)$-Pareto optimal at $n$.

Next we shall give two sufficient conditions for Condition $C(\mu)$ to be satisfied. For each $n \in N$ and $t \in T$, we define a stochastic process $\left(Y_{n}^{t}\right)$ by

$$
Y_{n}^{t}=X_{n}^{t}-E\left[X_{n+1}^{t} \mid \mathcal{F}_{n}\right]
$$

and a subset $A_{n}^{t}$ of $\Omega$ by

$$
A_{n}^{t}=\left\{Y_{n}^{t} \geq 0\right\} \equiv\left\{X_{n}^{t} \geq E\left[X_{n+1}^{t} \mid \mathcal{F}_{n}\right]\right\}
$$

Assumption 3.1. For $\mu \in \mathcal{H}$, there exists a subset $T_{0}$ of $T$ satisfying $\mu\left(T_{0}\right)=1$ such that for all $t \in T_{0}\left(Y_{n}^{t}\right)$ is a submartingale and $\lim _{n \rightarrow \infty} P\left(\cap_{t \in T_{0}} A_{n}^{t}\right)=1$.

When $\left(Y_{n}^{t}\right)$ is a submartingale, we have the relation

$$
E\left[X_{n+1}^{t} \mid \mathcal{F}_{n}\right] \geq \frac{1}{2} X_{n}^{t}+\frac{1}{2} E\left[X_{n+2}^{t} \mid \mathcal{F}_{n}\right]
$$


which means that $\left(X_{n}^{*}\right)$ is concave with regard to time parameter $n$. If $T_{0}$ is a finite set. $\left(A_{n}^{t}\right)$ is increasing with regard to $n$ and $\lim _{n \rightarrow \infty} P\left(A_{n}^{t}\right)=1$ for every $t \in T_{0}$, then $\lim _{n-x} P\left(\cap_{t \in T_{i}} A_{n}^{t}\right)=1$.

We define a new scalarized process $\left(Y_{n}(\mu)\right)$ by

$$
Y_{n}(\mu)=\int_{T} Y_{n}^{t} d \mu(t) \equiv X_{n}(\mu)-E\left[X_{n+1}(\mu) \mid \mathcal{F}_{n}\right]
$$

Then we notice that $A_{n}(\mu)=\left\{Y_{n}(\mu) \geq 0\right\}$.

LEMM1. 3.2. If Assumption 3.1 is satisfied for some $\mu \in \mathcal{M}$, then $\left(Y_{n}(\mu)\right)$ is a submartingale.

Proof. By the definition of $Y_{n}(\mu)$ and the fact that $\mu\left(T-T_{0}\right)=0$, it follow's that

$$
Y_{n}(\mu)-E\left[Y_{n+1}(\mu) \mid \mathcal{F}_{n}\right]=\int_{T_{1}}\left(Y_{n}^{t}-E\left[Y_{n+1}^{t} \mid \mathcal{F}_{n}\right]\right) d \mu(t) .
$$

Since $\left(Y_{n}^{t}\right)$ is a submartingale for any $t \in T_{0}$, that is, $Y_{n}^{t} \leq E\left[Y_{n+1}^{t} \mid \mathcal{F}_{n}\right], n \in N$, we have $Y_{n}(\mu) \leq E\left[Y_{n+1}(\mu) \mid \mathcal{F}_{n}\right]$ : and so $\left(Y_{n}(\mu)\right)$ is a submartingale for given $\mu \in \mathcal{M}$.

Theorem 3.3. If Assumption 3.1 is satisfied for some $\mu \in \mathcal{M}$, then Condition $C(\mu)$ holds, and hence for each $n \in N$ the $O L A$ rule $\sigma_{n}(\mu)$ is $(0, \mu)$-Pareto optimal at $n$.

Proof. In order to show that $A_{n}(\mu) \subset A_{n+1}(\mu)$, it suffices to prove that $X_{n+1}(\mu) \geq$ $E\left[X_{n+2}(\mu) \mid \mathcal{F}_{n+1}\right]$ on $A_{n}(\mu)$. Since $Y_{n}(\mu)$ is a submartingale from Lemma 3.2 and $Y_{n}(\mu) \geq 0$ on $A_{n}(\mu)$, it follows that for any $B \in \mathcal{F}_{n}$,

$$
\begin{aligned}
& \int_{A_{n}(\mu) \cap B}\left(X_{n+1}(\mu)-E\left[X_{n+2}(\mu) \mid \mathcal{F}_{n+1}\right]\right) d P \\
= & \int_{A_{n}(\mu) \cap B} Y_{n+1}(\mu) d P \\
\geq & \int_{A_{n}(\mu) \cap B} Y_{n}(\mu) d P \\
\geq & 0
\end{aligned}
$$

and hence $X_{n+1}(\mu) \geq E\left[X_{n+2}(\mu) \mid \mathcal{F}_{n+1}\right]$ on $A_{n}(\mu)$. Similarly, by using the fact that ( $\left.Y_{n}^{t}\right)$ is a submartingale for any $t \in T_{0}$, it follows that $A_{n}^{t} \subset A_{n+1}^{t}$ for every $n \in N$ and $t \in T_{0}$, so $\cap_{t \in T_{0}} A_{n}^{t} \subset \cap_{t \in T_{0}} A_{n+1}^{t}$ for every $n \in N$.

Next we see that for all $t \in T_{0} X_{n}^{t} \geq E\left[X_{n+1}^{t} \mid \mathcal{F}_{n}\right]$ on $n_{t \in T_{0}} A_{n}^{t}$. Since $\mu\left(T_{0}\right)=1$ for a. given $\mu \in \mathcal{M}$, it follows from Fubini's theorem for conditional expectation that

$$
\begin{aligned}
X_{n}(\mu) & =\int_{T_{0}} X_{n}^{t} d \mu(t) \\
& \geq \int_{T_{u \prime}} E\left[X_{n+1}^{t} \mid \mathcal{F}_{n}\right] d \mu(t) \\
& =E\left[\int_{T_{t}} X_{n+1}^{t} d \mu(t) \mid \mathcal{F}_{n}\right] \\
& =E\left[X_{n+1}(\mu) \mid \mathcal{F}_{n}\right]
\end{aligned}
$$


on $\cap_{t \in T_{v}} A_{n}^{t}$. Hence $\cap_{t \in T_{1}} A_{n}^{t} \subset A_{n}(\mu)$ and so $\cup_{n} \cap_{t \in T_{1 n}} A_{n}^{t} \subset U_{n} A_{n}(\mu)$. Since $P\left(\cup_{n} \cap_{T_{n}}\right.$ $\left.A_{n}^{t}\right)=\lim _{n-x} P\left(\cap_{t \in T_{1}}, A_{n}^{t}\right)=1$, we have $\lim _{n \rightarrow \infty} P\left(A_{n}(\mu)\right)=P\left(U_{n} A_{n}(\mu)\right)=1$. Thus Condition $C(\mu)$ is satisfied for a given $\mu \in \mathcal{M}$. From Theorem 3.1 it follows that the OLA rule $\sigma_{n}(\mu)$ is $(0, \mu)$-Pareto optimal at $n$.

Next we shall give another sufficient condition for Condition $C(\mu)$ to be satisfied.

Asslimption 3.2. For $\mu \in \mathcal{M}$, there exists a subset $T_{0}$ of $T$ satisfying $\mu\left(T_{0}\right)=$ 1 such that $A_{n}^{s}=A_{n}^{t}$ and $A_{n}^{t} \subset A_{n+1}^{t}$ for each $n \in N$ and any $s, t \in T_{0}$, and $\lim _{n \rightarrow \infty} P\left(A_{n}^{t}\right)=1$ for any $t \in T_{0}$.

Theorem 3.4. If Assumption 3.2 is satisfied for some $\mu \in \mathcal{M}$, then Condition $C(\mu)$ holds, and hence the OLA rule $\sigma_{n}(\mu)$ is $(0, \mu)$-Pareto optimal at $n$.

Proof. We see from Assumption 3.2 and by the way similar to the proof of Theorem 3.3 that $A_{n}^{t}=\cap_{s \in T_{0}} A_{n}^{s} \subset A_{n}(\mu)$ for each $n \in N$ and all $t \in T_{0}$. Conversely, since we have $X_{n}(\mu) \geq E\left[X_{n+1}(\mu) \mid \mathcal{F}_{n}\right]$ on $A_{n}(\mu)$, there exists $t \in T_{0}$ such that $X_{n}^{t} \geq E\left[X_{n+1}^{t} \mid \mathcal{F}_{n}\right]$, and so we have $A_{n}(\mu) \subset A_{n}^{t}=\cap_{s \in T_{u}} A_{n}^{s}$. Thus it follows that $A_{n}^{t}=\cap_{s \in T_{0}} A_{n}^{s}=A_{n}(\mu)$ for each $n \in N$ and all $t \in T_{0}$. From Assumption 3.2 the monotonicity of $\left\{A_{n}^{t}\right\}$ implies that of $\left\{A_{n}(\mu)\right\}$ and we have $\lim _{n \rightarrow \infty} P\left(A_{n}(\mu)\right)=1$, that is, Condition $C(\mu)$ is satisfied for a given $\mu \in \mathcal{M}$. Hence it follows from Theorem 3.1 that the OLA rule $\sigma_{n}(\mu)$ is $(0, \mu)$-Pareto optimal at $n$.

\section{Examples}

In this section we shall give four examples as applications of the previous section.

Example 4.1 (Withdrawal Problem I). Let $\left(W_{n}^{*}\right)$ be a random sequence which satisfies a regular condition, and assume that there is a subset $T_{0}$ of $T$ such that for each $t \in T_{0}$ and all $n \in N X_{n}^{t}=E\left[W_{n+a_{t}} \mid \mathcal{F}_{n}\right]$, where a parameter $a_{t}$ of time-shift is a nonnegative integer.

We define random sequence $\left(Z_{n}\right)$ and a subset $B_{n}$ of $\mathcal{F}_{n}$ by

$$
\begin{aligned}
& Z_{n}=W_{n}-E\left[W_{n+1} \mid \mathcal{F}_{n}\right], \\
& B_{n}=\left\{W_{n} \geq E\left[W_{n+1} \mid \mathcal{F}_{n}\right]\right\}=\left\{Z_{n} \geq 0\right\},
\end{aligned}
$$

respectively. We then have the relation $Y_{n}^{t}=E\left[Z_{n+a_{+}} \mid \mathcal{F}_{n}\right]$. We assume that $\left(Z_{n}\right)$ is a submartingale and $\lim _{n \rightarrow \infty} P\left(B_{n}\right)=1$. Here we notice that $\left(B_{n}\right)$ is an increasing sequence of sets. Then we easily see that $\left(Y_{n}^{t}\right)$ is a submartingale, so $A_{n}^{t} \subset A_{n+1}^{t}, n \in N$ for each $t \in T_{0}$. Also since $B_{n} \subset B_{n+a_{t}}$, we have $B_{n} \subset A_{n}^{t}$ for each $n \in N$ and all $t \in T_{0}$, so we have $\lim _{n \rightarrow x} P\left(\cap_{t \in T_{0}} A_{n}^{t}\right)=1$. Hence Assumption 3.1 is satisfied for any $\mu \in \mathcal{M}$ satisfying $\mu\left(T_{0}\right)=1$. Thus Theorem 3.3 implies that for such a $\mu$ the OLA rule $\sigma_{n}(\mu)$ is $(0, \mu)$-Pareto optimal at $n$ for every $n \in N$.

In a withdrawal problem from the market, we can explain this model as follows. There is a company which owns a lot of factories producing a common article at many 
countries in the world. Let $T$ be a set of all factories. $W_{n}$ represents demands for the article on LSA at time $n$ and is susceptible to the influences of the market. For a part of factories $T_{0}, X_{n}^{t}$ represents demands at the factory $t \in T_{0}$ and is influenced from $W_{n}$ with time delay $a_{t}$. The aim of the company is to find a withdrawal time $\tau$ from the market, at which all factories simultaneously stop to produce the article.

Example 4.2 (Withdrawal Problem II). For $t \in T$, let $f_{t}$ be a strictly increasing real-valued function on real line. For $\left(W_{n}\right)$ given in Example 4.1, there is a subset $T_{0}$ of $T$ such that for each $t \in T_{0}$ and all $n \in N X_{n}^{t}=f_{t}\left(W_{n}\right)$, for example, $X_{n}^{t}=a_{t} W_{n}+b_{t}$. where $a_{t}$ and $b_{t}$ are constants such that $a_{t}>0$. If $\left(W_{n}\right)$ is in monotone case, i.e. $B_{n} \subset B_{n+1}, n \in N$ and $\lim _{n \rightarrow \infty} P\left(B_{n}\right)=1$, we can easily check that Assumption 3.2 is satisfied. Thus it follows from Theorem 3.4 that for any $\mu \in \mathcal{M}$ satisfying $\mu\left(T_{0}\right)=1$, the OLA rule $\sigma_{n}(\mu)$ is $(0, \mu)$-Pareto optimal at $n$ for every $n \in N$. This model is also applicable to a withdrawal problem.

Example 4.3 (Withdrawal Problem III). For each $t \in T_{0} \subset T$, let $\left(U_{n}^{t}\right)$ be a bounded sequence of random variables defined on $(\Omega, \mathcal{F}, P)$ and adapted to $\left(\mathcal{F}_{n}\right)$. For a given constant $\beta_{t}\left(0<\beta_{t}<1\right)$ : discount factor, we define random sequence $\left(X_{n}^{t}\right)$ by

$$
X_{n}^{t}=\sum_{k=0}^{n} \beta_{t}^{k} U_{k}^{t},
$$

for $t \in T_{0}$. We assume that for each $t \in T_{0}$ and any $n \in N$

$$
\begin{aligned}
& E\left[U_{n+1}^{t} \mid U_{n}^{t}>0\right]>0, \\
& P\left(U_{n+1}^{t} \leq 0 \mid U_{n}^{t} \leq 0\right)=1
\end{aligned}
$$

and

$$
P\left(\cup_{n}\left\{U_{n}^{t} \leq 0\right\}\right)=1
$$

Then we easily see that $U_{n}^{t}>0$ if and only if $X_{n}^{t}<E\left[X_{n+1}^{t} \mid \mathcal{F}_{n}\right]$, and that if $U_{n}^{t} \leq 0$ then $X_{k}^{t} \geq E\left[X_{k+1}^{t} \mid \mathcal{F}_{k}\right\}$ for every $k \geq n$. Thus it follows that $A_{n}^{t}=\left\{U_{n}^{t} \leq 0\right\}, A_{n}^{t} \subset$ $A_{n+1}^{t}, t \in T_{0}, n \in N$ and $\lim _{n \rightarrow \infty} P\left(A_{n}^{t}\right)=1$.

Since

$$
Y_{n}^{\dagger}=-\beta_{t}^{n+1} E\left[U_{n+1}^{t} \mid \mathcal{F}_{n}\right]
$$

it follows that if

$$
E\left[U_{n+1}^{t} \mid \mathcal{F}_{n}\right] \geq \beta_{t} E\left[U_{n+2}^{t} \mid \mathcal{F}_{n}\right]
$$

for each $n \in N$ and any $t \in T_{0},\left(Y_{n}^{t}\right)$ is a submartingale, and hence that for any $\mu \in \mathcal{M}$ satisfying $\mu\left(T_{0}\right)=1$ Assumption 3.1 holds under condition that $\lim _{n \rightarrow \infty} P\left(\cap_{t \in T_{0}} A_{n}^{t}\right)=1$ or that $T_{0}$ is finite.

If $\left\{U_{n}^{s} \leq 0\right\}=\left\{U_{n}^{t} \leq 0\right\}$ for each $n \in N$ and any s.t $\in T_{0}$, Assumption 3.2 is satisfied for such a $\mu$.

We can explain this model as other withdrawal problem similarly to Huang and $\mathrm{Li}(1990)$ and Ohtsubo(1991). 
ExAmple 4.4 (CF. Chow et al.(1971)). For each $t \in T_{0} \subset T$, let $\left(W_{n}^{-t}\right)$ be a sequence of independent and identically distributed random variables with finite mean, and let

$$
m_{n}^{t}=\max _{0 \leq k \leq n} W_{k}^{t}, \quad X_{n}^{t}=m_{n}^{t}-c_{n}^{t}
$$

for each $n \in N$, where $\left(c_{n}^{t}\right)_{n=0}^{\infty}$ is any strictly increasing sequence of positive constants. Then we have

$$
X_{n+1}^{\mathrm{t}}-X_{n}^{t}=\left(W_{n+1}^{t}-m_{n}^{t}\right)^{+}-b_{n}^{t},
$$

where $b_{n}^{t}=c_{n+1}^{t}-c_{n}^{t}$. From Chow et al. $(1971, \mathrm{p} .56)$, it follows that if $b_{n+1}^{t} \geq b_{n}^{t}$ for all $n \in N$, that is, $\left(c_{n}^{t}\right)$ is convex with regard to $n$, then $A_{n}^{t} \subset A_{n+1}^{t}$ for any $n \in N$ and

$$
\lim _{n \rightarrow \infty} P\left(A_{n}^{t}\right)=P\left(\sigma_{t}<\infty\right)=1,
$$

where

$$
\sigma_{t}=\inf \left\{n \geq 0 \mid X_{n}^{t} \geq E\left[X_{n+1}^{t} \mid \mathcal{F}_{n}\right]\right\}=\inf \left\{n \geq 0 \mid m_{n}^{t} \geq \beta_{n}^{t}\right\}
$$

and $\beta_{n}^{t}$ is the unique solution of the equation

$$
E\left[\left(W_{n}^{t}-\beta_{n}^{t}\right)^{+}\right]=b_{n}^{t}
$$

Now in order to check that Assumption 3.1 is satisfied for any $\mu \in \mathcal{M}$ satisfying $\mu\left(T_{0}\right)=1$, it suffices to show that $\left(Y_{n}^{t}\right)$ is a submatingale. We have easily

$$
\begin{aligned}
E\left[Y_{n+1}^{t}-Y_{n}^{t} \mid \mathcal{F}_{n}\right] & =E\left[\left(X_{n+1}^{t}-X_{n+2}^{t}\right)-\left(X_{n}^{t}-X_{n+1}^{t}\right) \mid \mathcal{F}_{n}\right] \\
& =E\left[\left(W_{n+1}^{t}-m_{n}^{t}\right)^{+}-\left(W_{n+2}^{t}-m_{n+1}^{t}\right)^{+} \mid \mathcal{F}_{n}\right]-\left(b_{n}^{t}-b_{n+1}^{t}\right) .
\end{aligned}
$$

Since $m_{n}^{t} \leq m_{n+1}^{t}, n \in N$ and $\left(W_{n}^{t}\right)$ is i.i.d.,

$$
E\left[\left(W_{n+1}^{t}-m_{n}^{t}\right)^{+} \mid \mathcal{F}_{n}\right] \geq E\left[\left(W_{n+2}^{t}-m_{n+1}^{t}\right)^{+} \mid \mathcal{F}_{n}\right] .
$$

Thus when $\left(b_{n}^{t}\right)_{n=0}^{\infty}$ is an increasing sequence for every $t \in T_{0}$, it follows that $E\left[Y_{n+1}^{t} \mid \mathcal{F}_{n}\right] \geq$ $Y_{n}^{t}$, that is, $\left(Y_{n}^{t}\right)$ is a submatingale.

\section{References}

Aubin. J.P.(1979).Mathematical Methods of Game and Economic Theory, North-Holland, Amsterdam.

Chow, Y.S., Robbins H. and Siegmund D.(1971). Great Expectations : The Theory of Optimal Stopping, Houghton Miffin, Boston.

Gnedin, A.V.(1994). On a best-choice problem with dependent criteria, J. Appl. Prob. 31, 221-234.

Gugerli, L.S.(1987). Optimal stopping of a Markov chain with vector-valued gain function, Proc. 4th Vilnius Conference Prob. Theory Math. Statist, 2, VNU Sci. Press, Utrecht, 523-528. 
Hisano, H.(1980). An existence theorem in a rector-valued optimal stopping problem. Mem. Fac. Sci. Kyushu Univ., Ser.A 34. 99-106.

Huang, C.-F. and Li, L.(1990). Continuous time stopping games with monotone reward structures, Math. Operat. Res. 15, 496-507.

Neveu, J.(1975). Discrete-Parameter Martingales, North-Holland, Amsterdam.

Ohtsubo, Y.(1991). On a discrete-time non-zero-sum Dynkin problem with monotonicity, J. Appl. Prob. 28, 466-472.

Ohtsubo, Y.(1997). Multi-objective stopping problem for a monotone case, Mem. Fac. Sci. Kochi Univ. Ser.A 18, 99-104.

Preater, J.(1993). A note on monotonicity in optimal multiple stopping problems, Stat. Probab. Lett. 16, 407-410.

Stadje, W.(1980). Efficient stopping of a random series of partially ordered points, Springer Lecture Notes in Economics and Mathematical Systems. 177, 430-477.

Yu, P.-L.(1985). Multiple-criteria Decision Making, Plenum Press, New York.

Received October 9,1998

Revised April 23,1999 